\title{
A SUPPRESSED-ZERO ELECTRODYNAMIC VOLTMETER
}

\author{
By F. K. Harris
}

\begin{abstract}
An error is introduced into the reading of a deflecting instrument by inelastic yielding of the mechanism supplying the counter torque. This error, if large enough to affect precision, may be made negligible by keeping the instrument permanently deflected to one position and allowing only small changes in this deflection. For a voltmeter this procedure necessitates a variable multiplier which in turn introduces a special problem in temperature compensation. The present paper describes an attempt at the solution of these problems. The resulting instrument will measure alternating voltages between 20 and 300 volts at power frequencies to an accuracy of 0.01 per cent. An appendix discusses some difficulties encountered in obtaining a steady supply of alternating voltage and describes the methods used in eliminating the troubles.
\end{abstract}

\section{CONTENTS}

I. Introduction

II. Description

1. Instrument.

2. Optical system

3. Electrical circuits

4. Temperature compensation

III. Calibration

IV. Zero-drift error

V. Uses of the instrument

VI. Summary - -

VII. Appendix

1. Note on constant voltage supply

2. Note on d. c. supply for calibration of suppressed-zero voltmeter

\section{INTRODUCTION}

The voltmeter to be described was developed to meet the need for an a. c. instrument of greater precision and sensitivity than can be secured with available portable voltmeters. Specifically, an instrument was needed in our laboratory to measure voltages from 50 to 300 volts at power frequencies to an accuracy of 0.01 per cent. To meet these requirements, particular consideration must be given to two sources of error: (1) The temperature coefficent of the instrument must be either very accurately known or be made negligible; (2) the effect of inelastic yielding of the restoring springs must be eliminated. While this second source of error is usually negligible 
in the operation of deflecting instruments, it is always present, and in the case under consideration it is of primary importance.

In general, when any imperfectly elastic body is suddenly subjected to a load it suffers a certain deformation at once. Then, if the load is continuously applied, the deformation changes by an amount which is a function of the load applied, the duration of the load, and the previous load history of the body. ${ }^{1}$ In the case considered, when a constant voltage is impressed on a voltmeter the deflection of the moving element changes to some extent as long as the voltmeter remains in the circuit. When the circuit is opened, the pointer does not return at once to the zero position which it had before the application of the voltage, but to a position a little above it; that is, the effective zero point has moved upscale. The voltage reading will therefore be in error because of this zero shift which depends upon the length of time the voltmeter has been left in the circuit and to a lesser extent upon the previous readings taken with the instrument.

If this zero shift is proportional to the amount of the deflection, its effect can be made as small as desired by keeping the instrument always deflected to the point where it is to be used, by calibrating it in this condition, and by allowing only such changes in the deflection as are small in comparison to the total deflection. ${ }^{2}$

This has been done with the voltmeter described below. The moving system is mechanically held at a fixed position and is free to move through only a small angle. In order that the moving element may swing away from the stop against which it rests when no current is flowing, a definite value of current must pass through the instrument. To measure voltages, an adjustable resistance is connected in series with the instrument. The procedure followed is to vary the series resistance until the indicator swings freely between its stops. The impressed voltage is then known if the total resistance of the measuring circuit and the current required to produce the chosen deflection are known.

\section{DESCRIPTION}

\section{INSTRUMENT}

An electrodynamic voltmeter constructed in the Bureau of Standards instrument shop in 1908 was used in the set-up to be described. The moving element has two coils astatically arranged, each of 100 turns of silk-covered copper wire, No. 34 A. W. G. The moving element is suspended by phosphor-bronze strips between two sets of fixed coils mounted in wooden blocks. The fixed coils have about 460 turns each of copper wire, No. 30 A. W. G. These coils are con-

1 A. A. Michelson, Laws oi Elastico-viscous Flow, Proc. Nat. Acad. Sci., 3, pp. 319-323; 1917. M D. Hersey, On the Theory of Irreversible Time Effects, J. Wash. Acad. Sci., 11, pp. 149-155; 1921.

2 This procedure was suggested by Dr. II. B. Brooks. 
nected in series astatically and in series with the moving coils. The resistance of the six coils and the suspensions all in series is about 110 ohms. The inductance of the instrument is about 58 millihenrys.

A damping vane is attached to the moving system below the lower moving coil and swings with small clearance in a sector-shaped box.

A light aluminum arm is rigidly mounted on the moving system, and its motion is limited by two stops mounted on the case of the instrument. The moving element is free to rotate through an angle of slightly less than $3^{\circ}$ between the stops. The normal position of the moving element between the stops is such that the fixed coils and moving coils are very nearly at right angles to each other. Hence, the mutual inductance between the fixed coils and moving coils is nearly zero. In this instrument the rate of change of inductance with angle is a maximum for this position, and therefore the square law for the scale is closely followed for the small changes in angle allowed by the stops.

The free period of the instrument is about two seconds.

\section{OPTICAL SYSTEM}

For most purposes the mirror marked $C$ in Figure 1 is used with the usual arrangement of telescope and scale to indicate deflections. The scale is mounted at a distance of $6 \mathrm{~m}$ from theinstrument. It is $60 \mathrm{~cm}$ long and has its zero point in the center. The telescope is $4.5 \mathrm{~m}$ from the instrument. The entire free motion allowed in the moving system just sweeps the scale from end to end.

Another indicating system is used when further sensitivity is required. This is the double optical lever ${ }^{3}$ shown $_{\text {lere }}^{A}$ in Figure 2. The two mirrors marked $B$, moving mirror used with double $A, B$, in Figure 1 are used. One mirror ${ }_{C \text {, mirror used with scale and telescope. }}^{\text {optical lever }}$

${ }^{3}$ Attributed to Prof. L. Weber by H. Langner "Ueber eine Methode zur Messung kleiner Winkeldifferenzen," Zeits. für Instrumentenkunde, 6, pp. 299-304; 1886. 
is mounted on the case of the instrument and the other on the moving element. When these mirrors make an angle of about $90^{\circ}$ with each other, a beam of light from a pinhole source incident on the moving mirror is reflected to the fixed mirror and thence to the observer"s telescope. Another beam is reflected from the fixed mirror to the moving

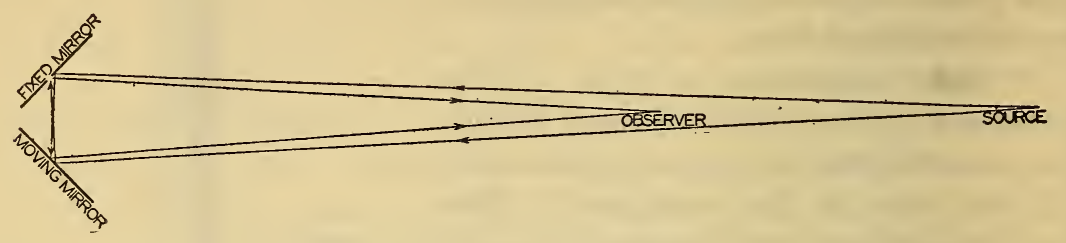

FIgure 2.-Double optical lever

mirror and to the telescope. Thus, two images of the source are seen in the telescope, and their approach or separation gives a fourfold magnification of the angular motion of the moving element. Their coincidence corresponds to the position of standard deflection.

The image of the scale appears in the observer's telescope just above these points of light and is always used for preliminary adjust-

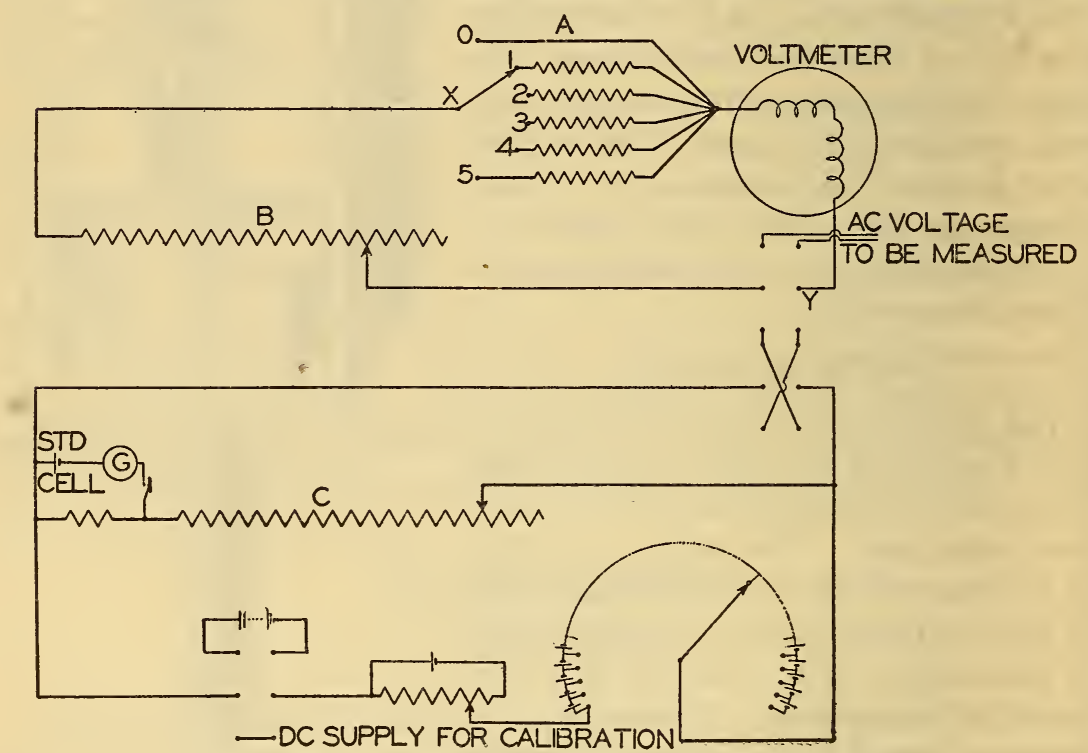

FigURE 3.-Schematic diagram of electrical circuits

ment of the deflection as well as for all settings where the greater sensitivity is not required.

\section{ELECTRICAL CIRCUITS}

A schematic diagram of the electrical circuits is shown in Figure 3. The main series resistor $B$, used in making all voltage readings, is varied until the moving system assumes the standard deflection 
described above. $B$ is a 6 -dial variable resistor of manganin wire so marked that one dial unit is 25 ohms.

When a calibration of theinstrument is desired, the equivalent potentiometer $C$ is thrown in parallel with the instrument and resistor $B$. The above-described standard deflection is then obtained simultaneously with a potentiometer balance on direct current.

The selector switch $X$ and the resistors $A$ are for temperature compensation.

\section{TEMPERATURE COMPENSATION}

In an electrodynamic milliammeter the torque is proportional to the square of the current and is balanced by the countertorque of the phosphor-bronze suspensions. Since the stiffness of the suspensions decreases approximately 0.04 per cent per degree centigrade increase of temperature, the reading will increase 0.02 per cent per degree. If the milliammeter plus added resistance is to be used as a voltmeter, this effect of temperature will be compensated if the resistance of the voltmeter increases 0.02 per cent per degree. Ordinarily this may be done by using a series resistor of negligible temperature coefficient having a resistance about nineteen times that of the copper coils. ${ }^{4}$

The suppressed-zero voltmeter, however, has two features requiring special consideration. The resistance of the measuring circuit is proportional to the voltage being measured. Hence, compensation merely by the addition of series resistance of negligible temperature coefficient is possible for only one value of voltage. Expansion and warping of the various parts of the instrument and its case with changing temperature may cause a shift of $(d D / d \theta) \delta \theta$ in the position of the suppressed zero. Because of this zero shift the temperature coefficient of the instrument can not be predicted, but must be measured.

Equating torques acting on the moving element for any temperature $\theta$

$$
\begin{aligned}
I^{2} d M / d \phi=U_{o}\left(1+\frac{d U / d \theta}{U_{o}} \delta \theta\right) D_{o}\left(1+\frac{d D / d \theta}{D_{o}} \delta \theta\right) \\
I=\sqrt{\frac{U_{o} D_{o}}{d M / d \phi}}\left(1+\frac{1}{2}\left[\frac{d U / d \theta}{U_{o}}+\frac{d D / d \theta}{D_{o}}\right] \delta \theta\right) \\
=K_{o}(1+\gamma \delta \theta)
\end{aligned}
$$

where

$U_{0}$ is the elastic constant of the suspensions at the temperature $\theta_{o}$,

$D_{o}$ is the deflection from the effective zero at $\theta_{0}$ to the standard position of the moving system,

$d M / d \phi$ is the rate of change of mutual inductance with angle,

$\gamma$ is the combined effect of the temperature coefficients of the elastic constant and the zero shift, and

$\delta \theta$ is the departure from the temperature $\theta_{o}$.

H. B. Brooks, The Accuracy of Commercial Electrical Measurements, Trans. A. I. E. E., 29, Pt. 1, p. $516 ; 1920$ 
Also the resistance of the circuit at any temperature is

where

$$
R=R_{E}(1+\alpha \delta \theta)+R_{\mathrm{S}}
$$

$\alpha$ is the temperature coefficient of resistance of copper,

$R_{E}$ is the resistance of the instrument at $\theta_{o}$, and

$R_{S}$ is the resistance of the series multiplier.

Consequently the voltage is, neglecting a second order term,

$$
V=I R=K_{o}\left(R_{E}+R_{S}\right)\left(1+\left[\frac{\alpha R_{E}}{R_{E}+R_{S}}+\gamma\right] \delta \theta\right)
$$

and the temperature coefficient of the instrument, defined as $\frac{1}{V} d V / d \theta$, is for this voltage

$$
\epsilon=\frac{\alpha R_{E}}{R_{E}+\bar{R}_{S}}+\gamma
$$

The compensation of the voltmeter here described is found to be perfect when about 10 per cent of the total resistance is of copper (at about 44 volts). Then, since $\alpha=0.4$ per cent, $\gamma=-0.04$ per cent. It should be noted that half of $\gamma$ is due to a shift of the effective zero with temperature.

It was desirable to have the instrument compensated for temperature throughout its useful range up to 250 volts. It was therefore necessary that the additional series resistance used in measurements above 44 volts have the correct temperature coefficient. If $\beta$ is the temperature coefficient of the added series resistance material $\left(R_{N}\right)$ used, then the temperature coefficient of the instrument becomes

$$
\epsilon=\frac{\alpha R_{E}+\beta R_{N}}{R_{E}+R_{N}+R_{S}}+\gamma
$$

Nickel wire was chosen for this purpose both because of its high resistivity and its large temperature coefficient, namely, 0.6 per cent per degree centigrade.

The final expression for the temperature coefficient of the instrument is then

$$
\epsilon=\frac{0.004 R_{E}+0.006 R_{N}}{R_{E}+R_{N}+R_{S}}-0.0004
$$

This compensating resistance should vary in temperature with the suspensions and coils of the instrument and should be insulated against outside temperature changes to the same degree. A single layer of nickel wire was therefore wound around the brass tubes which protect the suspensions. Half of this wire is wound around the lower suspension tube and half around the upper tube. It is protected against outside temperature changes by a layer of cotton batting which is in turn inclosed in brass tubes.

The suspensions, moving coils, fixed coils, and compensating resistance are thus all inclosed within the case of the instrument and are well 
insulated against sudden outside temperature changes. Their temperatures will, therefore, change very slowly and, under the conditions usually encountered in operation, will remain substantially equal.

The compensating resistance is wound in five sections which can be connected in the circuit by the dial switch $X$. (Fig. 3.) An additional constantan resistance is incorporated in each section to make the total resistance between points $(X)$ and $(Y)$ (fig. 3) $500 \mathrm{ohms}$ at $20^{\circ} \mathrm{C}$. for any position of the dial switch from 1 to 5 .

The positive temperature coefficient of resistance of the entire electrical circuit up to the selector switch $Y$ (fig. 3 ) must be equal to the combined effect of the zero shift and the change in stiffness of the suspensions for perfect compensation. For any particular resist-

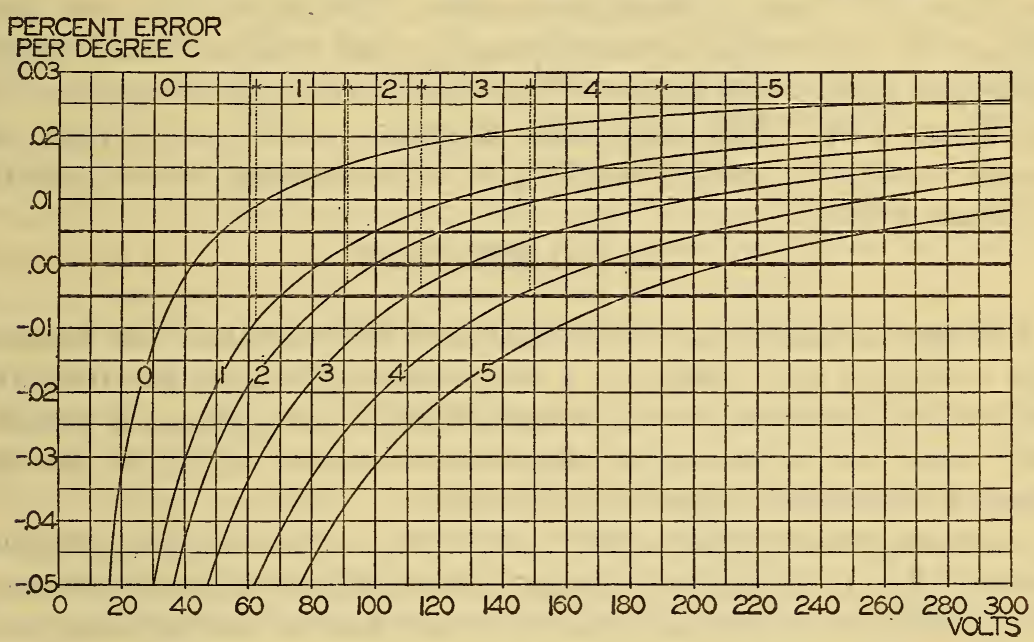

FIGURE 4.-Temperature compensation of voltmeter

Vertical dotted lines show voltage range through which each compensating unit (0-5, fig. 3) is to be used.

ance of nickel wire used there is only one value of total resistance (and one value of voltage) ${ }^{5}$ for which the two temperature coefficients are numerically equal. Then for this particular value of resistance and voltage the temperature coefficient of the instrument is zero. For smaller resistances and voltages the temperature coefficient will be negative and for larger values will be positive. For a considerable range it will be very small. By properly choosing a number of such points the temperature coefficient of the instrument can be made negligible throughout its useful range.

The actual value of the temperature coefficient of the instrument as a function of the voltage being measured is shown for the several dial settings in Figure 4. It will be seen from this figure that by

3 The voltage is measured by varying the total resistance of the instrument. 
proper selection of the compensating unit the temperature coefficient can be made less than 0.01 per cent per degree centigrade for voltages between 30 and 300 and less than 0.005 per cent per degree centigrade between 70 and 260 volts.

In view of the fact that calibration of the instrument is made frequently when accurate measurements are being made and since only such temperature changes as take place within the instrument case between the time of reading and the subsequent calibration affect the value of the reading, this small temperature coefficient is quite negligible.

The self-heating of the instrument is small. With the current chosen (0.04 ampere) to give the standard deflection, it amounts to less than $1^{\circ} \mathrm{C}$. for continuous operation. Neglecting the heat conduction to the terminals, the suspension must dissipate about 0.0025 watts per square centimeter. This should cause its temperature to rise about $1^{\circ} \mathrm{C}$. The coils must dissipate about 0.0008 watt per square centimeter, which should give a temperature rise of slightly less than $1^{\circ} \mathrm{C} .^{6}$

\section{CALIBRATION}

The current chosen to give the standard deflection was 0.04 ampere. The voltmeter has, therefore, a resistance of $25 \mathrm{ohms}$ per volt, and the voltage impressed on the terminals of the instrument is numerically equal to the reading of the dials of resistor $B$ (fig. 3 ) plus 20 , when the standard deflection is obtained.

In making the original calibration of the instrument the procedure was as follows: A value of voltage (100 volts) for which the temperature coefficient of the instrument is zero was set on the potentiometer $C$ (fig. 3), and resistor $B$ was set to read 80 dial units. Then, with the potentiometer balanced, the suspension clamps of the instrument were rotated until the moving system swung free between its mechanical stops and the standard deflection was indicated by the double optical lever (fig. 2). The suspension clamps were then tightened in this position.

When the voltage was removed from the instrument, the moving system rotated through $1.5^{\circ}$ and came to rest against the lower stop. The total rotation from the normal zero position of the moving system was about $60^{\circ}$.

In measuring a voltage it is only necessary to vary the resistance of resistor $B$ until the standard defiection is obtained. The voltage is then the reading of the dials of $B$ plus 20 .

\footnotetext{
- The value given in Whittaker's Electrical Engineer's Pocket Book, p. 111, 3d ed., for shellacked cotton bobbins is equivalent to 0.00086 watt per square centimeter per degree centigrade. The dissipation of 0.0025 watt per square centimeter per degree centigrade for the suspension seems reasonable on the basis of unpublished data at hand.
} 
When a more accurate value of voltage is required, the compensating dial $A$ (fig. 3 ) is set to the position which, for the voltage to be measured, most nearly reduces the temperature coefficient to zero. Resistor $B$ is next set in the usual manner. Then, without changing the setting of $B$, a direct voltage is substituted for the unknown alternating voltage and is varied until the standard deflection is again obtained. Holding this voltage constant, the potentiometer is then balanced. In order to correct for any lack of astaticism in the instrument, the above procedure is duplicated after reversing the direction of the direct current through the fixed coils and moving coils and the mean of the two potentiometer readings is taken.

The required value of alternating voltage differs from the mean of the potentiometer readings only in so far as the a. c. impedance of the instrument differs from its resistance. The error from this source is inversely proportional to the square of the voltage measured and amounts to 0.004 per cent at 100 volts and 60 cycles. It can, therefore, be considered negligible for most work.

A precise determination of voltage can be made in two or three minutes by an experienced operator. The determination can easily be made accurate to 0.01 per cent, and the sensitivity of the voltmeter is sufficiently great that differences in voltage as small as 0.002 per cent may be detected.

Where a number of successive readings are to be taken within the range of a volt or so, the following procedure has been found convenient. The voltmeter is calibrated with reversed direct voltage at some convenient value within the range. The change in voltage necessary to produce a deflection of $10 \mathrm{~cm}$ on the scale is determined. Then, with resistor $B$ set at the mean value found in the calibration, the deflection may be read for small changes in the voltage. The corresponding voltages are determined from the scale calibration just made. The scale calibration will vary with the voltage being measured, being about 0.01 volt per centimeter at 20 volts and 0.1 volt per centimeter at 200 volts.

\section{ZERO-DRIFT ERROR}

Drift measurements were made before the suppressed-zero arrangement was added to the instrument. A current of 0.046 ampere was passed through the coils and the sudden deflection ${ }^{7}$ was noted. The current was changed when necessary to maintain the deflection. The results of this test are shown in Figure 5.8 Equivalent drift

\footnotetext{
${ }^{7}$ By sudden deflection is meant the deflection which the moving system indicates as soon as it comes to rest after the voltage is impressed.

8 The data from which Figure 5 is plotted were supplied by Dr. F. B. Silsbee. The writer wishes to express his appreciation for this and for Doctor Silsbee's many helpful suggestions during the course of the investigation.
}

$64338^{\circ}-29-8$ 
is plotted as ordinates against time as abscissa. It may be noted that after the voltage has been impressed for five minutes the total drift is 0.04 per cent of the initial deflection. Hence, the rate of drift is approximately 0.01 per cent per minute initially. After onehalf hour the total drift is 0.2 per cent of the initial deflection. There

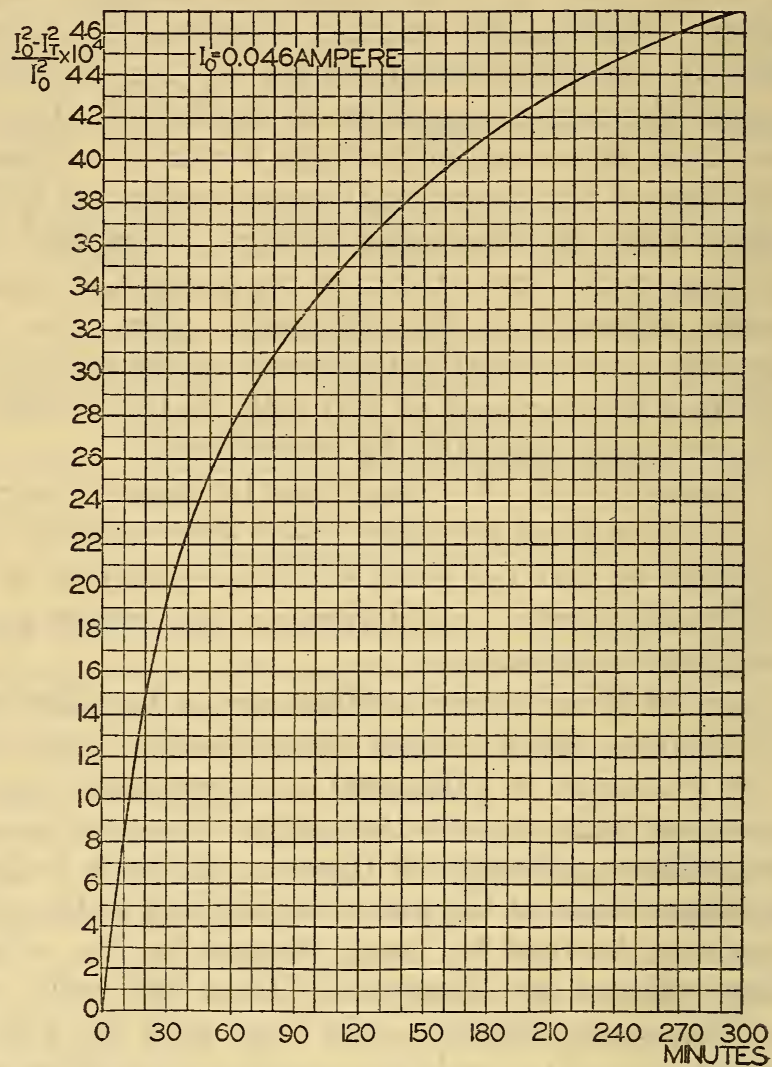

FiguRE 5.-Drift measurements starting from unstressed condition

The total change in deflection indicated is 0.47 per cent of the initial deflection represented by the point, $(0,0)$.

is no indication from the data taken that this drift ceases even after a number of days.

With the stops of the suppressed zero arrangement in place, the angular motion from the stop necessary to obtain the standard deflection is about 2 per cent of the sudden deflection on which Figure 5 has been based. If it be assumed that the drift is proportional to the amount of the sudden deflection, then the drift with the zero suppressed should be about 0.004 per cent during the first half hour after deflection takes place. 
A test of this argument was made recently after the moving system had been continuously deflected to within $1.5^{\circ}$ of its standard deflection $\left(60^{\circ}\right)$ for more than a year. A voltage was applied which brought the moving system from its mechanical lower stop to the standard deflection. This voltage was changed when necessary to maintain the standard deflection. The change in voltage was noted over a period of one-half hour from the time of its application. It amounted to about 0.003 per cent. This drift is nearly equivalent to the smallest change that can be detected with certainty and is entirely negligible. It can therefore be said that drift errors have been eliminated in the instrument.

An analysis was recently made of a large number of readings taken with the instrument over a period of 100 days. It showed a gradual and apparently uniform drift which amounted to 0.2 per cent for the whole period. In other words, the current required to produce the standard deflection was 0.2 per cent less at the end of the period than at the beginning.

Whether this difference is due to slipping of the suspension clamps, or to inelastic yielding in the suspensions or in the soldered joints at the suspension terminals, has not been determined. It seems probable, however, that the inelastic yielding, which amounts to a considerable item immediately after deflection, continues over a period of years at a very slow rate.

\section{USES OF THE INSTRUMENT}

The principal application of the instrument described above has been for the measurement of the secondary voltage of potential transformers. The primary voltage is then known as accurately as the ratio of transformation is known; that is, to within about 0.01 per cent at best. It has been found useful in various experiments where it was necessary to hold an alternating roltage constant within narrow limits, since it will detect and follow variations in voltage that can not be seen with the best portable instruments. ${ }^{9}$ It has also been used in the calibration of high-grade portable voltmeters.

The field of usefulness is restricted to precision measurements on account of the time consumed in making readings and the limited scale range of deflections. The scale range is about 3 per cent of the voltage measured, viz, 0.6 volt at 20 volts and 6 volts at 200 volts.

\section{SUMMARY}

An astatic electrodynamic voltmeter has been described in which errors arising from zero drift have been eliminated and whose temperature coefficient has been reduced to a negligible value. By using a

- See Appendir for note on constant alternating voltage supply. 
very long optical system to indicate deflections, differences of voltage as small as 0.002 per cent can be readily observed.

A d. c. potentiometer is built into the set-up so that the value of any alternating voltage within the range of the voltmeter can be readily determined in terms of the electromotive force of a standard cell. Measurements can be made on the instrument to an accuracy of 0.01 per cent for voltages between 20 and 300 volts.

\section{APPENDIX}

\section{NOTE ON CONSTANT VOLTAGE SUPPLY 10}

Considerable trouble has been experienced in obtaining alternating voltages which were sufficiently constant for precision work. The sensitivity available in the above-described instrument made it very useful for the study of this problem.

The source of a. c. supply studied was a direct-coupled motorgenerator set of two 60-cycle, $5 \mathrm{kv}$-a generators and a 250 -volt d. c. driving motor. The d. c. motor was supplied by a large storage battery.

In the course of this study a number of causes of trouble were found that could be practically eliminated. Since the voltmeter is sensitive to changes in supply voltage which may be produced by (1) variation in excitation, (2) variation in electrical load, and (3) variation in speed of the motor-generator set, a systematic investigation with this voltmeter disclosed the following undesirable conditions:

(a) Large variations in the exciting current in the rotating field windings were found to be a result of variations in brush contact resistance at the slip ring. Consequently, each brush was replaced by four smaller metal-graphite brushes with individual springs, operating independently in separate slots in the modified brush rigging.

(b) Variation in load was found to be caused in one case by a poorly-soldered stranded-wire connection of leads to the machine, in another by a loose fuse, and in another by an intermittent shorting of two turns of a large inductance coil.

(c) Variation in speed of the motor was found to be the result of variation of commutator-brush resistance (which was also improved by brush subdivision) and of variable friction in the bearings which was remedied by realignment and by replacement of bearings. Still further improvement resulted from driving the set by one generator operating as a synchronous motor in place of the d. c. motor and storage-battery drive. This synchronous motor was supplied from a 3 -phase generator of much greater moment of inertia, which in turn was driven by a d. c. motor supplied by the large storage battery.

${ }^{20}$ The work described in this section was done principally by Dr. F. M. Defandorf. 
It has been found necessary to give frequent attention to the slip rings in order to keep them as smooth and clean as possible. The subdivided brushes used are of the copper-graphite variety and show a smaller variation in contact resistance if additional lubricant is not used on the slip rings.

\section{NOTE ON DIRECT-CURRENT SUPPLY FOR CALIBRATION OF SUP- PRESSED-ZERO VOLTMETER}

For convenient calibration it is essential to have a source of direct voltage which is continuously variable within wide limits. This has been obtained as shown in Figure 3. A remotely controlled dial selector switch furnishes voltage in 10-volt steps up to 200 volts from a storage battery. In series with this supply is a smaller voltage ( 12 volts) connected to a rheostat of about 25 ohms resistance. The drop between one end and the slider of this rheostat provides for the fine adjustment necessary in making settings on the voltmeter. In case a higher voltage is needed another storage battery is introduced in the line in series with the other two.

This system was chosen for supplying the direct voltage for the instrument because the calibration is facilitated if the resistance of the d. c. source is low in comparison to the resistance of the set-up. It will be noted that, except for the fine adjustment which has a resistance of $2 \mathrm{ohms}$ per volt, the only resistance in the source is the internal resistance of the storage batteries used for supply and that of the leads to the instrument.

Washington, March 25, 1929. 